\title{
Avaliação de um periódico na área de medicina tropical*
}

\section{Sonia Pedrozo Gomes}

Bibliotecária chefe do Instituto de Medicina Tropical de São Paulo da Faculdade de Medicina da Universidade de São Paulo.

\section{Maria Aparecida de Lourdes Castro Santos}

Bibliotecária supervisora técnica de serviço do Serviço de Documentação e Divulgação Técnico-Científica do Sistema de Biblioteca e Documentação da Faculdade de Medicina da Universidade de São Paulo.

\begin{abstract}
Resumo
Realizou-se um estudo de avaliação da Revista do Instituto de Medicina Tropical de São Paulo, por meio de procedimentos de técnicas bibliométricas que identificaram qualidades e consistência científicas do periódico. Verificouse, mediante os resultados obtidos, que os conceitos de avaliação indicaram elevado desempenho do mesmo em relação aos seus aspectos intrínsecos e extrínsecos que permitiram conhecer: tipologia dos artigos publicados nas seções, temática enfocada para determinar o núcleo de assuntos de pesquisas realizadas e publicadas, procedência de autoria - nacional e estrangeira, vínculo autor/instituição, distribuição -, mala direta, identificação das bases de dados nas quais o periódico é indexado e freqüência de citação. Esses resultados oferecem importantes dados que podem contribuir significativamente como parâmetro para o estudo de avaliação de outros periódicos científicos.
\end{abstract}

\section{Palavras-chave}

Periódicos; Medicina tropical; Estudos de avaliação; Indicadores bibliométricos.

\section{Evaluation of a periodical in the tropical medicine area}

\begin{abstract}
An evaluation study of "Revista do Instituto de Medicina Tropical de São Paulo" was made using bibliometric analysis which have identified the scientific qualities and consistency of the periodical. Results indicated a significative performance in relation to its intrinsic and extrinsic aspects, namely: typology of the documents the sections, main research themes, origin of the authors (national and international), author-institution filiation, distribution (mailing list), data bases where the periodical is indexed and number of citations. These results offer important data that can contribute significantly as a parameter for evaluation studies of other scientific periodicals.
\end{abstract}

\section{Keywords}

Periodicals; Tropical medicine; Evaluation study; Bibliometric indicators.

\section{INTRODUÇÃO}

O interesse em avaliar a Revista do Instituto de Medicina Tropical de São Paulo deveu-se ao fato de que, durante toda a sua existência, nunca foi realizado um estudo bibliométrico capaz de mostrar suas características e qualidades e, ainda, sua repercussão em nível nacional e internacional, para atender aos objetivos de uma revista de disseminação científica.

Segundo Subramanyan apud Campello \& Campos** (1993), o periódico científico possui três funções: 1) registro público do conhecimento, pois qualquer indivíduo pode oferecer à apreciação um trabalho para publicação e, também, pode obter a publicação; 2) função social, isto é, ele atribui prestígio e reconhecimento tanto aos autores, quanto aos editores do periódico, aos referees e, inclusive, aos seus assinantes; 3) disseminação da informação, colocando a informação à disposição do leitor, pois, se ela não for publicada, não existe.

A avaliação dos periódicos pelas entidades internacionais depende mais do interesse particular delas do que da qualidade dos artigos contidos, isto é, se o assunto publicado não for "atraente", não gerará interesse na temática enfocada, principalmente se as doenças citadas incidem em determinadas regiões, restritas somente ao âmbito do país de origem ou aos países semelhantes. Todavia, esses periódicos são indispensáveis na disseminação da informação. Segundo Forattini (1996), para que um determinado periódico seja considerado indispensável à disseminação da informação, é necessário que ele, assim como os artigos nele contidos, possuam três atributos ou tríade: 1) competição (índice de citação); 2) impacto (alcance dos objetivos); 3) internacionalidade (indexação em órgãos especializados).

\footnotetext{
* Parte da monografia apresentada pelas alunas ao Curso de Especialização em Sistemas Automatizados de Informação Científica e Tecnológica para o Sistema Integrado de Bibliotecas da Universidade de São Paulo (SIBi/ USP) e Pontíficia Universidade Católica de Campinas (Puccamp), em 1999.

** SUBRAMANYAN, K. apud CAMPELlO, Bernadete Santos \& CAMPOS, Carlita Maria - Fontes de informação especializada: características e utilização. Belo Horizonte, Editora UFMG, 1993. p. $42-43$.
} 
Outro fato importante a ser destacado é que o periódico deve ser indexado em várias bases internacionais. Caso isso não aconteça, deve haver um "ciclo vicioso", como foi citado por Forattini (1996): "Se o artigo não é citado é porque foi publicado em periódico não indexado, este, por sua vez, deixa de sê-lo porque seus artigos não são citados". A citação de um trabalho publicado representa o reconhecimento de seu valor (Similowski \& Derenne, 1995).

\section{OBJETIVO GERAL}

- Avaliar a qualidade científica do periódico, mediante análises de conteúdo (mérito), forma (desempenho), sua citação em periódicos internacionais e autoria e, também, pela análise temática que mostra os assuntos das pesquisas realizadas e publicadas na Revista.

\section{OBJETIVOS ESPECÍFICOS}

- caracterizar os tipos de artigos publicados no período de 1994 a 1998;

- analisar e classificar a temática enfocada;

- identificar a procedência da autoria nacional e estrangeira nos artigos publicados;

- identificar os vínculos existentes entre autores/ instituições de São Paulo (capital);

- verificar a repercussão do periódico, através da sua mala direta, ano de 1998, localizando dentro dela o número de fascículos enviados ao exterior e Brasil;

- levantar as bases de dados onde a Revista do Instituto de Medicina Tropical de São Paulo está indexada;

- verificar a freqüência de citação do periódico, por intermédio de dois periódicos específicos da área.

\section{MATERIAL}

Por se tratar de uma pesquisa documental, o material analisado é um periódico científico da área médica, mais especificamente da área de medicina tropical.

A Revista do Instituto de Medicina Tropical de São Paulo, criada em 1959, é de responsabilidade do Instituto de Medicina Tropical de São Paulo, um centro de apoio com autonomia administrativa, diretamente ligado à Diretoria da Faculdade de Medicina da Universidade de São Paulo, direcionado ao apoio na pesquisa básica e laboratorial em doenças tropicais.
Desde a sua criação, a Revista do Instituto de Medicina Tropical de São Paulo é publicada bimestralmente, sem interrupção, sendo um dos mais antigos periódicos nacionais da especialidade, tendo completado em 1999, 40 anos de existência. A partir de 1997, passou a ser totalmente publicada na língua inglesa, obedecendo às normas técnicas exigidas e cronograma de publicação. Seu objetivo principal é a publicação de artigos relacionados à pesquisa em doenças tropicais e ciências afins.

Possui uma tiragem de 1.500 exemplares. É distribuída aos maiores centros de pesquisa, universidades, hospitais - nacionais e internacionais.

Tipos de literatura publicados: artigo original, carta ao editor, comunicação breve, editorial, ensaio técnico, ensaio terapêutico, homenagem, nota prévia, ponto de vista, recomendação técnica, relato de caso, relato técnico, revisão, revisão a convite, resumo de teses, resenha de livros.

Começou a disponibilizar, na Internet, por meio de sua home page, o conteúdo, a partir de 1996, de cada fascículo, site http://www.imtsp.fm.usp.br/rimtsp.htm.

A partir de 1997, foi integrada à Scientific Electronic Library Online (SciELO), podendo ser consultada integralmente no site http://www.scielo.br/rimtsp.

\section{MÉTODO}

Para a obtenção dos dados foram utilizados 30 fascículos do periódico Revista do Instituto de Medicina Tropical de São Paulo, abrangendo o período de 1994 a 1998, obedecendo aos procedimentos de análise a seguir:

1. A identificação dos assuntos mais publicados pelo periódico, no período, deu-se mediante leitura dos títulos e das análises dos resumos de 411 artigos $^{1}$. Houve a necessidade de se recorrer ao auxílio de um especialista atuante da área, como juiz, para que, em alguns artigos, fosse definido um de dois assuntos relevantes, quando esses de grande expressividade se confrontavam, pela opção de apenas um.

2. O tipo de literatura publicado foi coletado pela própria categoria temática dada pelo periódico, e foram tabulados fascículo por fascículo.

\footnotetext{
${ }^{1}$ Do total de 412 artigos, foi excluído um artigo - Carta ao Editor, por estar caracterizado como assunto de teor administrativo, e não biomédico.
} 
3. Identificou-se a autoria nacional e estrangeira (procedência), por intermédio das notas de rodapé, contidas nos artigos. Cabe destacar que foram colocados como "ñ indicados", na coluna localização geográfica, para aqueles autores que não foram identificados nas respectivas notas.

4. Tomando por base a identificação das autorias, analisaram-se os autores procedentes de São Paulo Capital (Vínculo Autor/Instituição), com a finalidade de saber qual o número exato de autores vinculados ao Instituto de Medicina Tropical de São Paulo, "autores da casa", que publicaram no período.

5. Foram analisados os locais de recebimento, por intermédio da distribuição da Revista do Instituto de Medicina Tropical de São Paulo, recorrendo-se à sua mala direta, relativa ao ano de 1998. Fez-se a identificação da distribuição, no exterior e nos estados do Brasil. Coletouse, também, a distribuição feita no interior de São Paulo.

6. Foram pesquisadas, no período de 1994 a 1998, as bases em que a Revista do Instituto de Medicina Tropical de São Paulo estava indexada.

7. Verificou-se a freqüência de citação do periódico Revista do Instituto de Medicina Tropical de São Paulo, como indicador de qualidade. Tomamos como base a análise de citação em dois periódicos internacionais, publicados em 1998, da área de medicina tropical, considerados de renome internacional ${ }^{2}$. Foram identificados os três autores nacionais mais citados nesses periódicos.

\section{RESULTADOS E DISCUSSÃO}

Para melhor compreensão dos resultados, os dados foram reunidos em algumas tabelas, quadros e figuras.

O número total de artigos publicados no período de 1994 a 1998 foi de 412, o que resulta em uma média anual de 13,73 artigos por fascículo. Considerando-se as seções de resenha de livros, resumo de teses e video review, o número total de tipos de literatura publicados, nesse período, passa a ser de 484 .

\footnotetext{
${ }^{2}$ Os periódicos escolhidos para a análise de citação tiveram a aprovação do editor adjunto.
}

\section{TABELA 1}

Tipos de artigos publicados de 1994 a 1998

\begin{tabular}{|c|c|c|c|c|c|c|}
\hline TIPOS DE & & & ANOS & & & TOTAL \\
\hline ARTIGOS & 1994 & 1995 & 1996 & 1997 & 1998 & \\
\hline Artigos originais & 65 & 69 & 54 & 43 & 50 & 281 \\
\hline Comunicação breve & 8 & 12 & 10 & 8 & 11 & 49 \\
\hline Relato de caso & 8 & 8 & 4 & 8 & 9 & 37 \\
\hline Carta ao editor & 3 & 4 & 1 & 2 & - & 10 \\
\hline Ensaios técnicos & 3 & - & 2 & 1 & - & 6 \\
\hline Nota prévia & 1 & 1 & 1 & 2 & 1 & 6 \\
\hline Revisão & 1 & 1 & - & 3 & 1 & 6 \\
\hline Revisão a convite & - & 1 & 2 & 1 & 1 & 5 \\
\hline Ensaios terapêuticos & - & - & 2 & - & 1 & 3 \\
\hline Homenagem & 1 & - & - & 1 & 1 & 3 \\
\hline Editorial & - & - & 1 & 1 & - & 2 \\
\hline Bioética & 1 & - & - & - & - & 1 \\
\hline Ponto de vista & 1 & - & - & - & - & 1 \\
\hline Recomendações técnicas & - & - & 1 & - & - & 1 \\
\hline Relato técnico & - & - & - & - & 1 & 1 \\
\hline TOTAL & 92 & 96 & 78 & 70 & 76 & 412 \\
\hline
\end{tabular}

\section{Tipos de artigos publicados}

Comparando a tipologia dos artigos publicados, as seções que mais se destacaram foram artigos originais com 281 (68,20\%), seguidos por comunicação breve 49 (11,90\%), relato de caso $37(8,99 \%)$, carta ao editor $10(2,43 \%)$, revisão, nota prévia e ensaios técnicos $6(1,45 \%)$ cada, revisão a convite $5(1,21 \%)$, homenagem e ensaios terapêuticos $3(0,73 \%)$ cada, editorial $2(0,5 \%)$ e, finalmente, relato técnico, recomendações técnicas, ponto de vista e bioética, com 01 artigo cada (0,24\%) (tabela 1).

\section{Assuntos mais publicados}

A medicina tropical é um dos segmentos da medicina que se ocupa das doenças prevalentes nas regiões tropicais e subtropicais (Rey, 1999).

Segundo Forattini (1997), a expressão medicina tropical teve sua origem no sistema colonialista, tratando-se de uma denominação consagrada pelo uso e empregada corretamente para os problemas nosológicos de regiões com predomínio do macroclima tropical e subtropical.

Deve-se destacar, também, que vários outros fatores contribuem na problemática das doenças endêmicas, emergentes e ressurgentes, as quais são encontradas em todo o território do Brasil, fazendo-se o Brasil ser constituído de vários "Brasis", como, por exemplo, a região Sul é considerada como próxima a país desenvolvido (Forattini, 1997).

A análise temática dos assuntos dos artigos publicados, no período de 1994 a 1998, deu-se do maior para os de menor participação, porém não de menor valor de consistência, resultando em 13 áreas temáticas. Essas áreas 
temáticas foram identificadas pela leitura dos títulos e análise de conteúdo dos resumos, confrontando os termos mais gerais no DeCS (Descritores em Ciências da Saúde) e submetendo-os à apreciação de um especialista da área de doenças infecciosas e parasitárias, apresentadas na tabela 2 .

Houve a predominância nas áreas de parasitologia e epidemiologia. Destacou-se a área de parasitologia com um resultado de 30,18\%, no período, devendo-se provavelmente à falta de periódico desta área em nível internacional que possibilite a maior difusão de dados parasitológicos obtidos neste país.

Em segundo lugar, vem a área de epidemiologia, com o resultado de $24,10 \%$, no período, devendo provavelmente essa participação à necessidade de conhecimento da incidência das diversas moléstias infecciosas no país.

Observamos que, no terceiro lugar, está presente a área de micologia, com um resultado de $12,41 \%$, devendo-se provavelmente este fato ao grande número de trabalhos micológicos que advém de laboratórios no país de nível internacional, localizados principalmente nos estados de São Paulo, Rio de Janeiro e Minas Gerais.

Em quarto lugar, está presente a área de imunologia com uma participação de $11,92 \%$, que é fundamental para a compreensão dos mecanismos patogenéticos em doenças infecciosas.

As áreas de microbiologia e virologia, ambas com o mesmo resultado $8,76 \%$, vêm em quinto lugar, com aspecto compreensível dada à importância dessas áreas no conjunto de doenças infecciosas.

\section{Procedência de autoria nacional e estrangeira}

Na tabela 3, obteve-se o total de 2.029 autores nacionais e estrangeiros, distribuídos em 1.648 (81,19\%) autores nacionais, $346(17,04 \%)$ autores estrangeiros e 35 (1,77\%) autores que não foi possível identificar o local de origem, porque as notas de rodapé estavam incompletas.

Da autoria nacional, verificou-se que as dez maiores procedências foram dos estados de São Paulo, com 865, seguido de Minas Gerais (264), Rio de Janeiro (196), Rio Grande do Sul (60), Pernambuco (43), Góias (37), Pará (32), Bahia (30), Paraná (23) e Ceará (22). Analisandose a autoria nacional por grandes regiões, temos o seguinte: Norte (47), Nordeste (108), Sudeste (1331), Sul (98) e Centro-Oeste (64).
TABELA 2

Classificação dos assuntos publicados no período de 1994 a 1998

\begin{tabular}{lcccccc}
\hline \multicolumn{1}{c}{ ASSUNTOS } & 1994 & 1995 & 1996 & 1997 & 1998 & TOTAL \\
Alergia & 3 & 0 & 0 & 2 & 1 & 6 \\
Bioética & 1 & 0 & 0 & 0 & 0 & 1 \\
Biologia molecular & 0 & 0 & 0 & 1 & 0 & 1 \\
Dermatologia & 1 & 0 & 0 & 0 & 0 & 1 \\
Epidemiologia & 21 & 24 & 24 & 11 & 19 & 99 \\
Hematologia & 1 & 0 & 2 & 0 & 0 & 3 \\
Imunologia & 15 & 15 & 7 & 7 & 5 & 49 \\
Micologia & 14 & 11 & 7 & 11 & 8 & 51 \\
Microbiologia & 8 & 10 & 11 & 2 & 5 & 36 \\
Nutrição & 0 & 1 & 1 & 0 & 0 & 2 \\
Parasitologia & 27 & 27 & 21 & 27 & 22 & 124 \\
Toxicologia & 0 & 1 & 1 & 0 & 0 & 2 \\
Virologia & 1 & 6 & 4 & 9 & 16 & 36 \\
TOTAL & 92 & 95 & 78 & 70 & 76 & 411 \\
\hline
\end{tabular}

\section{TABELA 3}

Totalização dos autores por área de procedência

\begin{tabular}{|c|c|c|c|c|c|c|}
\hline LOCALIZAÇÃO & & & ANOS & & & TOTAL \\
\hline GEOGRÁFICA & 1994 & 1995 & 1996 & 1997 & 1998 & \\
\hline BRASIL & 338 & 395 & 294 & 310 & 311 & 1648 \\
\hline EXTERIOR & 97 & 71 & 86 & 33 & 59 & 346 \\
\hline Não indicados & 13 & 4 & 14 & 2 & 3 & 35 \\
\hline TOTAL GERAL & 448 & 470 & 394 & 345 & 373 & 2029 \\
\hline
\end{tabular}

A predominância da região Sudeste vem indicar a concentração de grandes institutos de pesquisas e centros de ensino superior, onde há maior produtividade de trabalhos científicos, como Instituto Oswaldo Cruz (RJ), Instituto de Ciências Biológicas da UFMG (MG), Instituto de Medicina Tropical de São Paulo, Faculdade de Medicina da USP e Escola Paulista de Medicina (SP).

Verificou-se que as dez maiores procedências de autoria do exterior são Argentina (88), Venezuela (35), Cuba (32), Uruguai (30), Colômbia (28), Estados Unidos (26), Peru (19), Japão (18), Chile (15) e Bélgica (10).

\section{Vínculo Autor/Instituição}

Dos autores de procedência São Paulo Capital (SPC), em um total de 690 (34\%), foram analisados novamente um a um por intermédio das notas de rodapé, considerando-se o vínculo autor/instituição para constatar se houve freqüente inclusão de autores institucionais, segundo Castro et alii (1996a) "Sabe-se que a inclusão sistemática de alta porcentagem de artigos de autores institucionais prejudica os periódicos que almejam indexação em bases de dados internacionais". 
Verificou-se, analisando os resultados da filiação dos autores, que essa informação nem sempre é bem colocada, incompleta, prejudicando demasiadamente a localização institucional dos autores, pois aparece por siglas, não mantendo uma uniformidade.

No gráfico 1, foram identificadas as dez instituições que mais se destacaram por meio dos vínculos autores/ instituições.

Da observação dos resultados nessa figura, constata-se um fator extremamente relevante: os autores em questão tiveram o maior número de procedência do Instituto Adolfo Lutz e Instituto de Medicina Tropical de São Paulo. Convém salientar que esse aspecto deve-se à integração das duas instituições, por haver semelhanças das áreas de interesse na pesquisa e, também, proximidade geográfica das mesmas. Apesar de ambas terem importância equivalente, nota-se que o vínculo autores/instituições está refletido em outras instituições que possuem interesse semelhante de pesquisa em grupo na área de doenças infecciosas e parasitárias.

\section{Análise da distribuição (mala direta)}

A distribuição de uma revista científica precisa ser adequada, porque é através da boa divulgação que se fazem o reconhecimento e o prestígio do periódico e dos autores nela contidos (Valerio, 1994).

A Revista do Instituto de Medicina Tropical de São Paulo possui uma tiragem de 1.500 exemplares, é distribuída pela própria administração da Revista por doação, permuta e assinatura. Deve-se destacar que a Biblioteca do Instituto de Medicina Tropical de São Paulo, criada em 15 de janeiro de 1959, é mantida desde essa data com as permutas efetuadas entre a Revista do Instituto de Medicina Tropical de São Paulo com os outros periódicos nacionais e internacionais, pois a Biblioteca não dispõe de verba para compor o seu acervo. As assinaturas não são suficientes para o seu custeio. Durante o ano de 1998, foram efetuadas 118 assinaturas para o Brasil e sete assinaturas para o exterior.

Analisando a mala direta de 1998, constatamos que a circulação do periódico concentra-se principalmente em âmbito internacional. Os dados aqui mostrados foram calculados incluindo a doação, a permuta e a assinatura.

\section{GRÁFICO 1}

Vínculos de autores/instituições, destacando-se os dez maiores

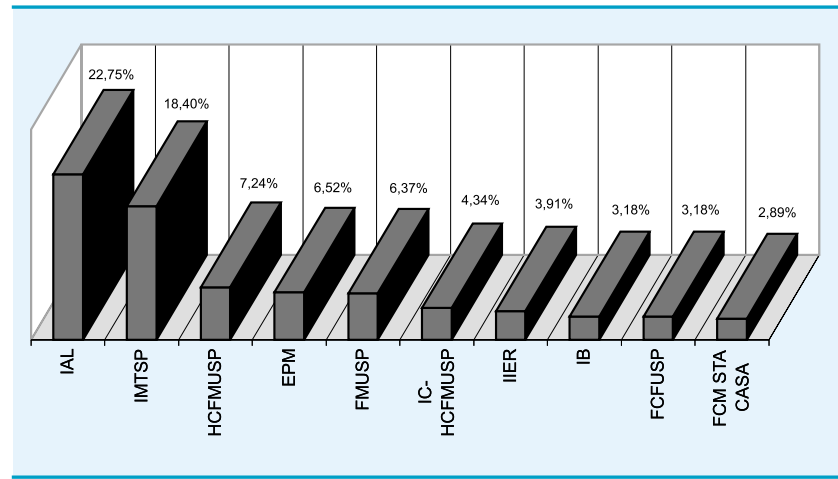

\section{QUADRO 1}

Distribuição mundial, em ordem alfabética de países" , da Revista do Instituto de Medicina Tropical de São Paulo

\begin{tabular}{|c|c|c|c|}
\hline Alemanha & 12 & Itália & 15 \\
\hline Angola & 1 & Iuguslávia & \\
\hline Argélia & 1 & Jamaica & \\
\hline Argentina & 45 & Japão & 18 \\
\hline Austrália & 11 & Libéria & \\
\hline Áustria & 2 & Luxemburgo & \\
\hline Bolívia & 14 & Madagascar & \\
\hline Bulgária & 10 & Malásia & \\
\hline Canadá & 4 & Marrocos & \\
\hline Checoslováquia & 6 & México & 34 \\
\hline Chile & 15 & Moçambique & \\
\hline China & 12 & Nicarágua & \\
\hline Colômbia & 32 & Nigéria & \\
\hline Coréia & 1 & Noruega & \\
\hline Costa Rica & 10 & Nova Guiné & \\
\hline Cuba & 11 & Nova Zelândia & \\
\hline Dinamarca & 2 & Panamá & \\
\hline Egito & 5 & Paraguai & \\
\hline El Salvador & 3 & Pasquistão & \\
\hline Equador & 13 & Peru & 30 \\
\hline Espanha & 11 & Polônia & 4 \\
\hline Estados Unidos & 91 & Porto Rico & \\
\hline Etiópia & 1 & Portugal & \\
\hline Filândia & 1 & Quênia & \\
\hline Filipinas & 5 & República Dominicana & \\
\hline França & 24 & Romênia & \\
\hline Ghana & 1 & Rússia & \\
\hline Grã-Bretanha & 40 & Senegal & 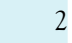 \\
\hline Grécia & 1 & Suécia & \\
\hline Guatemala & 5 & Suíça & 12 \\
\hline Guiana Francesa & 1 & Suriname & 2 \\
\hline Guiné-Bissau & 1 & Tailândia & 1 \\
\hline Haiti & 2 & Tunísia & \\
\hline Holanda & 5 & Turquia & \\
\hline Honduras & 2 & Uganda & 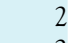 \\
\hline Hungria & 2 & União Sul Africana & 2 \\
\hline Índia & 12 & Uruguai & 12 \\
\hline Irã & 3 & Venezuela & 20 \\
\hline Irlanda & 1 & Zimbabwe & 1 \\
\hline Israel & 2 & Total & 640 \\
\hline
\end{tabular}

* Os nomes dos países foram seguidos à risca, conforme encontrados na mala direta cedida pela administração da Revista. 
Entre as dez maiores economias do mundo, segundo Seidl (1999), medidas pelo Produto Interno Bruto (PIB), estão Estados Unidos, Japão, Alemanha, França, Reino Unido, Itália e China. Esses países são fundamentais, também, no processo de comunicação da ciência, e a Revista do Instituto de Medicina Tropical de São Paulo faz parte dessa comunidade científica.

Pode-se afirmar que o referido periódico conseguiu alcançar a meta de distribuição internacional, pois pode ser encontrado em vários países do mundo, conforme distribuição efetuada por continentes na tabela 4 .

No Brasil, a circulação total é de 431 revistas, sendo 119 no estado de São Paulo. Desse total, 43 encontram-se em cidades do interior de São Paulo, tabela 5.

A região Sudeste destaca-se como o maior canal de circulação do periódico, com 232 exemplares recebidos (tabela 6).

\section{Indexação nas bases de dados}

Lemos (1981) fez uma análise sobre indexação de revistas brasileiras no exterior, para comprovar que nossos periódicos não são totalmente desconhecidos da comunidade científica internacional. Nesse estudo, pode ser visto que, se essas revistas mantêm os padrões essenciais de qualidade científica, podem ser indexadas por essas bases. Como foi o caso da Revista do Instituto de Medicina Tropical de São Paulo, que, segundo Brennen \& Davey apud Lemos (1981), fazia parte dos 41 títulos da área de medicina tropical indexados pelo Tropical Diseases Bulletin e que atingiu, nessa época, o décimo segundo lugar, com 173 citações.

É extremamente complicado ter acesso às publicações dos trabalhos científicos, divulgados nos quatro cantos do mundo, dificultando sobremaneira uma cobertura adequadamente completa. Entretanto, para incluir ou excluir periódicos em um índice de literatura científica são abordados vários critérios de avaliação. O Institute for Scientific Information define um núcleo essencial de periódicos para suas bases de dados que abrangem todas as áreas do conhecimento. O núcleo é avaliado periodicamente e pode ser modificado, portanto a exclusão e/ou a inclusão de determinados títulos de periódicos é feita constantemente (Testa, 1998).

Em 1985, a Revista do Instituto de Medicina Tropical de São Paulo fazia parte desse núcleo essencial de periódicos, na área de medicina tropical, ocupando o nono lugar. E, dentro dessa mesma área, ocupava o vigésimo lugar em artigos citados, internacionalmente (Roelants, 1987).
TABELA 4

Distribuição mundial da Revista do Instituto de Medicina Tropical de São Paulo, por continentes

\begin{tabular}{lc}
\hline Continentes & Total \\
África & 033 \\
América Central & 044 \\
América do Norte & 129 \\
América do Sul & 188 \\
Ásia & 051 \\
Europa & 188 \\
Oceania & 013 \\
Total & 640 \\
\hline
\end{tabular}

\section{TABELA 5}

Distribuição da Revista do Instituto de Medicina Tropical de São Paulo, por Estados da Federação

\begin{tabular}{lr}
\hline Estados & Total \\
Acre & 02 \\
Alagoas & 07 \\
Amazonas & 07 \\
Bahia & 22 \\
Ceará & 02 \\
Distrito Federal & 15 \\
Espírito Santo & 07 \\
Goiás & 12 \\
Maranhão & 14 \\
Mato Grosso & 04 \\
Mato Grosso do Sul & 05 \\
Minas Gerais & 50 \\
Pará & 16 \\
Paraíba & 12 \\
Paraná & 20 \\
Pernambuco & 20 \\
Piauí & 03 \\
Rio de Janeiro & 431 \\
Rio Grande do Norte & 56 \\
Rio Grande do Sul & 119 \\
Rondônia & 05 \\
Santa Catarina & 15 \\
São Paulo & 03 \\
Sergipe & 11 \\
Total & \\
\hline & \\
\hline
\end{tabular}

TABELA 6

Distribuição da Revista do Instituto de Medicina Tropical de São Paulo, por grandes regióes

\begin{tabular}{lc}
\hline Regiões & Total \\
Centro-Oeste & 036 \\
Norte & 028 \\
Nordeste & 089 \\
Sul & 046 \\
Sudeste & 232 \\
Total & 431
\end{tabular}


Em 1993, a Revista do Instituto de Medicina Tropical de São Paulo foi excluída do "Current Contents" e do "Science Citation Index", por não atender a dois critérios exigidos pelas instituições mencionadas, a saber: os artigos redigidos em inglês e a periodicidade. Gaillard apud Sayão* (1996) constatou que, nas grandes bases internacionais, encontram-se incluídos somente $5 \%$ da produção científica dos países do Terceiro Mundo. Com isso, a afirmação de Miranda (1996) ilustra qual é a finalidade dos trabalhos científicos: "a do publique ou pereça e a sua contraparte, seja citado ou desapareça".

As bases de dados e índices impressos onde a Revista do Instituto de Medicina Tropical de São Paulo está indexada são as seguintes: AidsLine, Biosis Previews, Cab Abstracts, Cab Health, Cancerlit, Embase - Excerpta Medica, Helminthological Abstracts, Hepatologie, Index Veterinarium, Lilacs6, MedWebPlus, Medline/Index Medicus/PubMed (são a mesma base), Periódica - Índice de Revista Latinoamericana en Ciencias, Protozoological Abstracts, Review of Medical and Veterinary Entomology, Review of Medical and Veterinary Mycology, SciELO (a Revista do Instituto de Medicina Tropical de São Paulo contribui, em relação a essa base de dados, com uma média de participação significativa de 11,39\% de artigos por fascículos, conforme "Relatórios de Citações de Revistas" disponíveis no site http://www.scielo.br), SciSearch, Tropical Diseases Bulletin, Veterinary Bulletin, UnCoverWeb.

Segundo Braga \& Oberhofer (1982), em seu modelo para avaliação de periódicos científicos e técnicos, procura mostrar os aspectos de forma de um periódico, por meio de parâmetros mensuráveis. A indexação é definida como "um indicador de julgamentos de qualidade já consolidados; assim, quanto mais numerosa a inclusão em serviços de indexação, maior a disseminação; como a maioria desses serviços é seletiva, a inclusão do periódico reflete um reconhecimento positivo da qualidade dos artigos".

Krzyzanowski \& Ferreira (1998) também avaliaram esse critério e constataram que a indexação do periódico em bases de dados internacionais faz com que ele receba uma valorização de qualidade e produtividade, isso significa que quanto maior o número de indexação maior será sua abrangência.

Como conseqüência dessa análise, constatou-se que a Revista do Instituto de Medicina Tropical de São Paulo obteve

* GAILLARD, Jacques apud SAYÃO, L.F. Bases de dados: a metáfora da memória científica. Ciência da Informação, Brasília, v. 25, n. 3, 1996. Disponível em: < http://www.ibict.br/cionline/> Acesso em: 26 jan. 1999. um nível de desempenho muito bom, pois a inclusão do periódico nesses serviços de indexação citados vem indicar o nível de qualidade que ela possui.

Hoje, ela vem lutando para conseguir a sua aceitabilidade no Institute for Scientific Information (ISI), instituição que desde a década de 70 vem trabalhando com afinco nesse setor. Esse Instituto é responsável por várias publicações, tais como Current Contents (CC), Science Citation Index (SCI) e outras, considerado pelo professor Forattini, editor da Revista de Saúde Pública da Universidade de São Paulo, como o "Olimpo da divulgação científica". A Revista do Instituto de Medicina Tropical de São Paulo vem tentando, através de mudanças em seu conteúdo, reingressar a indexação no ISI.

Como citado por Castro et alii (1996b), os periódicos indexados em bases internacionais são subsidiados pelas agências governamentais que apóiam a pesquisa para o seu financiamento. Esta afirmação pode ser constatada na Revista do Instituto de Medicina Tropical de São Paulo, que recebe subsídios de várias Instituições, como Fapesp, CNPq, SIBi/USP e Fundação Faculdade de Medicina/USP (FFM/USP).

\section{Freqüência de citação do periódico}

As referências bibliográficas contidas em um determinado trabalho auxiliam na avaliação da informação coletada, pois permitem ao pesquisador o conhecimento de outros trabalhos que tratam do mesmo tema, isto é, direcionando-o para outras fontes de informação. A análise das citações utilizadas nesses trabalhos permite avaliar qual o tipo de literatura utilizada, assim como ajudar no reconhecimento pessoal do pesquisador (Noronha, 1998). Acrescenta-se, ainda, que a análise de citação permite conhecer, segundo Mello, 1996, "os dados referentes à quantidade e à identificação da literatura de uma área da ciência”, e, segundo Similowski \& Derenne (1995), a citação de um trabalho publicado representa o reconhecimento de seu valor.

Foi utilizada a análise das citações como uma técnica cientométrica ${ }^{3}$, tomando por base o número de citações que o periódico analisado recebeu da comunidade científica selecionada, através de dois periódicos internacionais da área, o American Journal of Tropical Medicine and Hygiene e o Annals of Tropical Medicine and Parasitology.

\footnotetext{
${ }^{3}$ Cientometria é a especialidade da sociologia da ciência que compreende todos os tipos de análises quantitativas da ciência que se baseiam em fontes de arquivo, sem observação direta da atividade de pesquisa (Mello, 1996)
} 
Os artigos citados nos periódicos anteriormente mencionados foram classificados por:

1. Procedência de autoria, isto é, qual o país do(s) autor(es) que escreveu/escreveram o artigo publicado nas revistas selecionadas e citou/citaram a Revista do Instituto de Medicina Tropical de São Paulo.

2. Temporalidade das citações da Revista do Instituto de Medicina Tropical de São Paulo nos documentos citados.

3. Autoria dos artigos da Revista do Instituto de Medicina Tropical de São Paulo mais citados nos periódicos selecionados.

\section{- American Journal of Tropical Medicine and Hygiene (AJTMH)}

Responsabilidade da American Society of Tropical Medicine and Hygiene, Estados Unidos, fundado em 1921, no ano de 1998 completou 77 anos de publicação contínua. Esse periódico é indexado no Index Medicus, Biological Abstracts, Protozoological Abstracts, Helminthological Abstracts, Review of Applied Entomology, Current Contents (Life Sciences) e Tropical Diseases Bulletin. Publica dois volumes por ano, a periodicidade é mensal e a circulação é de 4.400 exemplares. Home page http:// www.ajtmh.org. ISSN: 0002-9637. O número de referências bibliográficas analisadas nos 327 artigos publicados, em 1998, foi de 8.505. Dessas referências, 59 (0,7\%) eram da Revista do Instituto de Medicina Tropical de São Paulo.

As tabelas 7 e 8 descrevem as citações encontradas nos dois volumes analisados.

A temporalidade das citações foi medida em períodos de dez em dez anos, com exceção do último período que vai de 1990 a 1997. Pode se verificar, na tabela 9, a seguir, que a maior concentração das citações da Revista do Instituto de Medicina Tropical de São Paulo ocorreu no período de 1990 a 1997, com 25 citações.

A maior procedência geográfica dos autores deu-se no Brasil, com 38 vezes. Nesse mesmo sentido, verificamse os Estados Unidos, como o segundo país, com 17 vezes, a citar a Revista do Instituto de Medicina Tropical de São Paulo. Seguidos pela Argentina (6), Bolívia e Japão (4), Colômbia (3), Chile / Espanha / Holanda / Irlanda / Suíça (2), Bélgica / China / França / Grã-Bretanha / Paraguai / Peru / Porto Rico / Quênia / Venezuela e Zimbabwe (1).
TABELA 7

American Journal of Tropical Medicine and Hygiene volume 58,1998

\begin{tabular}{|c|c|c|c|c|}
\hline FASC. & $\begin{array}{l}\text { PÁG. } \\
\text { INIC. }\end{array}$ & $\begin{array}{l}\text { PROCEDÊNCIA DO(S) } \\
\text { AUTOR(ES) no AJTMH }\end{array}$ & \multicolumn{2}{|c|}{$\begin{array}{c}\text { AUTOR(ES) CITADOS DA } \\
\text { REVISTA DO IMTSP - REFERENCIA COMPLETA }\end{array}$} \\
\hline 1 & 7 & Brasil & SCHALL, VT. et al. & 34: 183-191, 1992 \\
\hline 1 & 7 & Brasil & SOUZA, CP. et al. & 34: 459-466, 1992 \\
\hline 1 & 26 & Brasil & KATZ, N. et al. & 14: 397-400, 1972 \\
\hline 1 & 50 & Bolívia / Espanha & KATZ, N. et al. & 14: 397-402, 1972 \\
\hline 1 & 122 & Zimbabwe & MONTOYA, F. et al. & 36: 33-38, 1994 \\
\hline 2 & 183 & Colômbia / Brasil & CUPO, P. et al. & 33: 115-122, 1991 \\
\hline 3 & 338 & Venezuela & $\begin{array}{l}\text { BRANDÃO, HJS \& } \\
\text { ZULIAN, R. }\end{array}$ & $8: 281-286,1966$ \\
\hline 4 & 417 & Irlanda / Bolívia & KATZ, N. et al. & 14: 397-402, 1972 \\
\hline 4 & 417 & Irlanda / Bolívia & $\begin{array}{l}\text { VAZ, AJ \& FERREIRA, } \\
\text { AW. }\end{array}$ & 30: $1-10,1988$ \\
\hline 4 & 424 & Colômbia / Estados Unidos & CAMPOS, EP et al. & 33: 267-276, 1991 \\
\hline 4 & 448 & Brasil / Grã-Bretanha / Suiça & $\begin{array}{l}\text { DEANE, LM \& DEANE, } \\
\text { MP. }\end{array}$ & 4: 198-212, 1962 \\
\hline 4 & 505 & Brasil / Japão & NAIFF, RD. et al. & $28: 19-27,1986$ \\
\hline 4 & 505 & Brasil / Japão & MARQUES, SA. et al. & $25: 87-92,1983$ \\
\hline 4 & 505 & Brasil / Japão & VIDAL, MS. et al. & $37: 43-49,1995$ \\
\hline 5 & 630 & Brasil / Estados Unidos & ANDRADE, JG. et al. & 34: 467-473, 1992 \\
\hline 5 & 630 & Brasil / Estados Unidos & DI SANTI, SM. et al. & 30: $147-152,1988$ \\
\hline 6 & 693 & Peru / Estados Unidos & VAZ, AJ. et al. & $32: 355-359,1990$ \\
\hline 6 & 694 & Brasil & VAZ, AJ. et al. & 38: 391-396, 1996 \\
\hline 6 & 694 & Brasil & FERREIRA, AP. et al. & 39: 29-33, 1997 \\
\hline 6 & 694 & Brasil & ANDRADE, APF. et al. & 38: 259-263, 1996 \\
\hline 6 & 748 & Argentina / Estados Unidos & STEINDEL, M. et al. & $36: 43-50,1994$ \\
\hline 6 & 748 & Argentina / Estados Unidos & MARSDEN, PD. et al. & $21: 13-25,1979$ \\
\hline 6 & 807 & Brasil / Estados Unidos & COURA, JR. et al. & 37: 415-420, 1995 \\
\hline 6 & 824 & Brasil & CHIARI, CA. et al. & $15: 298-303,1973$ \\
\hline 6 & 824 & Brasil & MARZOCHI, MCA. et al. & $55: 149-155,1980(*)$ \\
\hline 6 & 824 & Brasil & MAYRINK, W. et al. & 48: $182-185,1976(* *)$ \\
\hline 6 & 824 & Brasil & MAYRINK, W. et al. & $55: 565-569,1983(* * *)$ \\
\hline 6 & 828 & Estados Unidos / Japão & GRENAN, MM. et al. & 27: 190-196, 1985 \\
\hline
\end{tabular}

(*) Referência incorreta, o certo é 22: 149-155, 1980

(**) Referência incorreta, o certo é 18: 182-185, 1976.

(***) Referência incorreta, o certo é 25: 265-269, 1983.

\section{TABELA 8}

American Journal of Tropical Medicine and Hygiene volume 59,1998

\begin{tabular}{|c|c|c|c|c|}
\hline \multirow{2}{*}{$\begin{array}{c}\text { FASC. } \\
1\end{array}$} & \multirow{2}{*}{$\begin{array}{l}\text { PÁG. } \\
\text { INIC. } \\
53\end{array}$} & \multirow{2}{*}{$\begin{array}{l}\text { PROCEDÊNCIA DO(S) } \\
\text { AUTOR(ES) no AJTMH } \\
\text { Estados Unidos / Brasil }\end{array}$} & \multicolumn{2}{|c|}{$\begin{array}{c}\text { AUTOR(ES) CITADOS DA } \\
\text { REVISTA DO IMTSP - REFERENCIA COMPLETA }\end{array}$} \\
\hline & & & ALENCAR, JE. & 3: $175-180,1961$ \\
\hline 1 & 66 & Estados Unidos / Brasil & $\begin{array}{l}\text { SHIKANAI-YASUDA, } \\
\text { MA. et al. }\end{array}$ & $34: 85-90,1992$ \\
\hline 1 & 124 & Colômbia / Estados Unidos & ALECRIM, MG. et al. & 24: 44-47, 1982 \\
\hline 1 & 133 & Chile / Argentina & CAMARGO, M. & $8: 227-234,1966$ \\
\hline 1 & 133 & Chile / Argentina & LEVI, GC. et al. & $38: 35-38,1996$ \\
\hline 1 & 150 & Holanda & KATZ, N. et al. & 14: $397-400,1972$ \\
\hline 2 & 325 & Brasil & TADEI, WP. et al. & $30: 221-251,1988$ \\
\hline 3 & 357 & Brasil & FIGUEIREDO, LTM. et al. & 34: 121-130, 1992 \\
\hline 3 & 357 & Brasil & $\begin{array}{l}\text { VASCONCELOS, PFC. et } \\
\text { al. }\end{array}$ & $35: 141-148,1993$ \\
\hline 3 & 357 & Brasil & OSANAI, CH. et al. & 23: 53-54, 1983 \\
\hline 3 & 357 & Brasil & $\begin{array}{l}\text { VASCONCELOS, PFC. et } \\
\text { al. }\end{array}$ & $37: 253-255,1995$ \\
\hline 3 & 370 & China / Holanda / Bélgica & KATZ, N. et al. & 14: 397-400, 1972 \\
\hline 3 & 487 & Paraguai & BITTENCOURT, AL. & $34: 403-408,1992$ \\
\hline 3 & 492 & Brasil & SALGADO, JA. et al. & $4: 330-337,1962$ \\
\hline 4 & 530 & Estados Unidos / Brasil / Suíça & CAMARGO, ME. et al. & 26: 192-204, 1984 \\
\hline 4 & 543 & Brasil & CUBA-CUBA, CA. et al. & 33: 343-350, 1991 \\
\hline 4 & 557 & Brasil & $\begin{array}{l}\text { ANDRADE, ZA \& } \\
\text { ANDRADE, SG. }\end{array}$ & $7: 218-227,1965$ \\
\hline 4 & 563 & Estados Unidos / Brasil & CAMARGO, ME. & 6: 93-100, 1964 \\
\hline 4 & 644 & $\begin{array}{l}\text { Quênia / Estados Unidos / Porto } \\
\text { Rico }\end{array}$ & NASSAR, ES. et al. & 37: 337-341, 1995 \\
\hline 5 & 679 & Brasil & LEAL, NC. et al. & $31: 295-300,1989$ \\
\hline 5 & 736 & Brasil & GUIMARÃES, ACS. et al. & $35: 479-483,1993$ \\
\hline 5 & 736 & Brasil & OSTERMAYER, AL. et al. & $38: 328,1996$ \\
\hline 5 & 736 & Brasil & $\begin{array}{l}\text { CAMARGO, ME \& } \\
\text { LESER, PG }\end{array}$ & $18: 227-238,1976$ \\
\hline 5 & 741 & Argentina / Estados Unidos & MARSDEN, PD. et al. & $24: 364-373,1982$ \\
\hline 5 & 741 & Argentina / Estados Unidos & LAURICELLA, MA. et al. & $31: 63-70,1989$ \\
\hline 5 & 750 & Brasil & REICHE, EMV. et al. & $38: 233-240,1996$ \\
\hline 5 & 750 & Brasil & CAMARGO, ME. & $8: 227-234,1966$ \\
\hline 5 & 750 & Brasil & CAMARGO, ME. & 6: 93-100, 1964 \\
\hline 5 & 750 & Brasil & SILVA, AMM. et al. & 31: 146-150, 1989 \\
\hline 5 & 784 & Brasil / França & PITTELLA, JEH. & 35: 111-116, 1993 \\
\hline 6 & 922 & Espanha / Bolívia & KATZ, N. et al. & $14: 397-402,1972$ \\
\hline
\end{tabular}




\section{- Annals of Tropical Medicine and Parasitology (ATMP)}

Publicado pela Liverpool School of Tropical Medicine, Grã-Bretanha, desde 1907. É editada oito vezes ao ano, nos meses de janeiro, março, abril, junho, julho, setembro, outubro e dezembro. Está indexada nas seguintes bases de dados CAB Abstracts, CAB Health, Chemical Abstracts, Current Contents (Life Sciences e Clinical Medicine), Excerpta Medica/Embase, Index Medicus/Medline, Pascal, Research Alert, Science Citation Index, SciSearch e Tropical Diseases Bulletin. Home page http:// www.carfax.co.uk/atm-atm-ad.htm. ISSN: 0003-4983.

Foram analisadas 2.417 referências bibliográficas, em 104 artigos publicados durante o ano de 1998, no referido periódico, sendo encontradas 7 (0,29\%) citações da Revista do Instituto de Medicina Tropical de São Paulo.

A tabela 10 indica as citações encontradas nessa revista. Em relação às revistas internacionais analisadas (American Journal of Tropical Medicine and Hygiene e Annals of Tropical Medicine and Parasitology), a Revista do Instituto de Medicina Tropical de São Paulo apresenta alto índice de citação na primeira, porém nem tanto na segunda, conforme tabelas 7,8 e 10.

Na tabela 11, é medida a temporalidade de citação, em espaços de 10 anos, para 1970/1979 e 1980/1989, e de 3 anos, para 1990/1993, considerando para esse último a mais recente data citada, com a finalidade de se fazer conhecer o período mais citado. Destacou-se o período de 1990 a 1993, com três citações da Revista do Instituto de Medicina Tropical de São Paulo.

A análise das citações da Revista do Instituto de Medicina Tropical de São Paulo mostrou que o período mais citado, nos dois periódicos selecionados durante o ano de 1998, foi de 1990 a 1997, com 28 (42,42\%) citações, de um total de 66 .

A procedência geográfica de autoria para os três primeiros países citados totalizou-se em: Brasil (38), Estados Unidos (17) e Argentina (6).

Os três autores mais citados da Revista do Instituto de Medicina Tropical de São Paulo de um total de 66 citações, considerando-se somente o primeiro autor, foram o primeiro de Minas Gerais, com sete citações, o segundo de São Paulo, com seis, e o terceiro, também, de São Paulo, com três citações.
TABELA 9

Período mais citado da Revista do Instituto de Medicina Tropical de São Paulo no American Journal of Tropical Medicine and Hygiene

Anos

Total

$1960-1969$

1970-1979

1980-1989

1990-1997

Total

\section{TABELA 10}

Annals of Tropical Medicine and Parasitology volume 92, 1998

\begin{tabular}{cclll}
\hline Fasc. & Pág. inic. & $\begin{array}{r}\text { Procedência do(s) autores } \\
\text { do ATMP }\end{array}$ & \multicolumn{2}{c}{$\begin{array}{r}\text { Autor(es) citados da } \\
\text { revista do IMTSP - referência completa }\end{array}$} \\
2 & 219 & França / Bolívia & $\begin{array}{l}\text { CARVALHEIRO, JR. \& } \\
\text { BARRETTO, MP. }\end{array}$ & $18: 17-23,1976$ \\
2 & 213 & Brasil & JORGE, MT. et alii & $32: 6-10,1990$ \\
2 & 213 & Brasil & ANDRADE, JG. et alii & $31: 363-367,1989$ \\
6 & 671 & Argentina & LAURICELLA, MA. et alii $31: 63-70,1989$ \\
7 & 765 & Austrália / China & LI, YS. et alii & $35: 347-353,1993$ \\
7 & 765 & Austrália / China & KATZ, N. et alii & $14: 397-400,1972$ \\
8 & 895 & Venezuela & MEDRANO, C. et alii & $34: 63-71,1992$ \\
\hline
\end{tabular}

\section{TABELA 11}

Período mais citado da Revista do Instituto de Medicina Tropical de São Paulo no Annals of Tropical Medicine and Parasitology

\begin{tabular}{ll}
\hline Anos & Total \\
$1970-1979$ & 2 \\
$1980-1989$ & 2 \\
$1990-1993$ & 3 \\
Total & 7 \\
\hline
\end{tabular}

Os demais autores não considerados ficaram empatados, no quarto lugar, com duas citações cada.

\section{CONCLUSÃO E SUGESTÕES}

A avaliação realizada não esgotou a possibilidade de se fazerem outros estudos, enfocando vários aspectos do periódico, que poderão fornecer importantes dados que estariam contribuindo significativamente no seu aprimoramento.

Tomando-se por base alguns indicadores extrínsecos e intrínsecos da Revista do Instituto de Medicina Tropical de São Paulo, podemos dizer que os resultados apresentados puderam destacar o elevado desempenho do referido periódico. 
- Circulação: é maior em âmbito internacional, alcançando a credibilidade e o prestígio das agências financiadoras.

- Indexação: o periódico encontrava-se indexado em vários serviços especializados, e houve novas descobertas no período analisado (1994/1998).

- Assuntos publicados: pela análise dos assuntos, os resultados obtidos indicaram o referido periódico como pertencente ao núcleo de revistas mais refinadas da área, atingindo altos percentuais na publicação de artigos relacionados à pesquisa em doenças infecciosas e parasitárias e ciências afins.

- Tipologia dos artigos publicados: a distribuição do conteúdo dos artigos teve como destaque os artigos originais, com 68,20\%, publicados no período estudado (1994/1998).

- Origem de autoria: quanto aos aspectos de autoria nacional, verificou-se a predominância de autores dos estados de São Paulo, Minas Gerais e Rio de Janeiro, destacando-se a região Sudeste como a maior região em produtividade científica, mas salientando-se que não houve o predomínio de autores domésticos. Quanto à autoria estrangeira, destacou-se o maior número de autores da Argentina, Venezuela, Cuba, Uruguai e Colômbia, sobretudo no período em que a Revista aceitava artigos em espanhol e português. A partir do não-recebimento de artigos nessas línguas, e sim, em inglês, houve significante aumento da participação de autores procedentes dos Estados Unidos.

Concluímos que os conceitos avaliados pela escala de valoração publicada no artigo de Krzyzanowski \& Ferreira (1998) mostram que a Revista do Instituto de Medicina Tropical de São Paulo contém qualidade e consistência científica que a levarão à sua indexação em mais bases de dados renomadas, tanto nacionais quanto internacionais, possibilitando melhor disseminação das pesquisas realizadas nesta área e, também, servindo como parâmetro para estudo de avaliação de outros periódicos científicos.

\section{AGRADECIMENTOS}

Às professoras Cecília Carmen Cunha Pontes, Rosaly Favero Krzyzanowski, coordenadoras e orientadoras do Curso de Especialização em Sistemas Automatizados de Informação Científica e Tecnológica para o SIBi/USP Puccamp, à Regina Célia Figueiredo Castro, pela leitura e revisão do texto, e à professora Maria de Cléofas Faggion Alencar, pela ajuda na finalização do presente artigo.

\section{REFERÊNCIAS BIBLIOGRÁFICAS}

BIREME (São Paulo, SP). Descritores em ciências da saúde: lista alfabética. 2. ed. rev. ampl. São Paulo, 1992.

BRAGA, Gilda Maria; OBERHOFER, Cecília Alves. Diretrizes para a avaliação de periódicos científicos e técnicos brasileiros. Revista Latinoamericana de Documentación, v. 2, n. 1, p. 27-31, 1982.

BRENNEN, Patrick W. \& DAVEY, W. Patrick apud LEMOS, Antonio A. Briquet. Infra-estrutura da literatura biomédica: considerações acerca de um núcleo de revistas brasileiras do setor saúde. Educación. Médica y Salud, v. 15, n. 4, p 406-423, 1981.

CASTRO, Regina Célia Figueiredo; FERREIRA, Maria Cecília Gonzaga; VIDILI, A. L. Periódicos latino-americanos: avaliação das características formais e sua relação com a qualidade científica. Ciência da Informação, Brasília, v. 25, n. 3, p. 357-367, 1996a.

CASTRO, Regina Célia Figueiredo; NEGRÃO, May Brooking; ZAHER, Célia Ribeiro. Procedimentos editoriais na avaliação de artigos para publicação em periódicos de ciências da saúde da América Latina e Caribe. Ciência da Informação, Brasília, v. 25, p. 3, 1996b. Disponível em: <http://www.ibict.br/cionline/> Acesso em: 26 jan. 1999.

FORATTINI, Oswaldo Paulo. A tríade da publicação científica. Revista. de Saúde Pública., v. 30, n. 1, p. 3-12, 1996.

FORATTINI, Oswaldo Paulo. O Brasil e a medicina tropical. Revista de Saúde Pública, v.31, n. 2, p. 116-120, 1997.

GAILLARD, Jacques SAYÃO, L.F. Bases de dados: a metáfora da memória científica. Ciência da Informação, Brasília, v. 25, n. 3, 1996. Disponível em: <http://www.ibict.br/cionline/> Acesso em: 26 jan. 1999.

KRZYZANOWSKI, Rosaly Favero; FERREIRA, Maria Cecília Gonzaga. Avaliação de periódicos científicos e técnicos brasileiros. Ciência da Informação, Brasília, v. 27, n. 2, p. 165-175, 1998.

LEMOS, Antonio A. Briquet. Infra-estrutura da literatura biomédica: considerações acerca de um núcleo de revistas brasileiras do setor saúde. Educación Médica y Salud, v. 15, n. 4, p. 406-423, 1981.

MELLO, Paula Maria Abrantes Cotta de. A citação bibliográfica no contexto da comunicação: um estudo exploratório na área da botânica. Ciência da Informação, Brasília, v. 25, n. 3, 1996. Disponível em: < http:/ /www.ibict.br/cionline> Acesso em: 26 jan. 1999.

MIRANDA, Dely Bezerra de. O períodico científico como veículo de comunicação: uma revisão de literatura. Ciência da Informação, Brasília, v. 24, n. , 1996. Disponível em: < http://www.ibict.br/cionline> Acesso em: 26 jan. 1999.

NORONHA, Daisy Pires. Análise das citações das dissertações de mestrado e teses de doutorado em saúde pública (1990-1994): estudo exploratório. Ciência da. Informação, Brasília, v. 27, n. 1, p. 66-75, 1998.

REY, Luís. Dicionário de termos técnicos de medicina e saúde. Rio de Janeiro: Guanabara Koogan, 1999.

ROELANTS, G. - Citation analysis in the field of tropical medicine. Ann. Soc. Belge Med.. trop., v. 67, n. 4, p. 315-318, 1987.

SEIDL, Antonio Carlos. Brasil perde o 8 o lugar no ranking mundial. Folha de São Paulo, São Paulo, 15 abr. 1999. Caderno Dinheiro, p. 12.

SIMILOWSKI, T.; DERENNE, J. P. Bibliométrie des périodiques biomédicaux. Rev. Mal. resp., v. 12, p. 543-550, 1995.

SUBRAMANYAN, K. CAMPELLO, Bernadete Santos; CAMPOS, Carlita Maria. Fontes de informação especializada: características e utilização. Belo Horizonte, Editora UFMG, 1993. p. 42-43.

TESTA, James. A base de dados ISI e seu processo de seleção de revistas. Ciência da Informação, Brasília, v. 27, n. 2, p. 233-235, 1998.

VALÉRIO, Palmira Moriconi. Espelho da ciência: avaliação do programa setorial de publicações em ciência e tecnologia da FINEP. Brasília : FINEP/IBICT, 1994. 